\title{
Fast Antimicrobial Susceptibility Testing on Escherichia coli by Metabolic Heat Nanocalorimetry
}

Received 00th January 20xx Accepted 00th January 20xx DOI: $10.1039 / x 0 x \times 00000 x$

\begin{abstract}
Yang Liu ${ }^{a}$, Thomas Lehnert ${ }^{\mathrm{a}}$ and Martin A. M. Gijs*a
Fast spreading of antimicrobial resistance is now considered a major global health threat. New technologies are required, enabling rapid diagnostics of bacterial infection combined with fast antimicrobial susceptibility testing (AST) for evaluating the efficiency and dosage of antimicrobial compounds in vitro. This work presents an integrated chip-based isothermal nanocalorimetry platform for direct microbial metabolic heat measurements and evaluates its potential for fast AST. Direct detection of the bacteria-generated heat allows monitoring of metabolic activity and antimicrobial action at subinhibitory concentrations in real-time. The high heat sensitivity of the platform enables bacterial growth detection within only a few hours of incubation, whereas growth inhibition upon administration of antibiotics is revealed by a decrease or the absence of the heat signal. Antimicrobial stress results in lag phase extension and metabolic energy spilling. Oxygen consumption and optical density measurements provide a more holistic insight of the metabolic state and the evolution of bacterial biomass. As a proof-of-concept, a metabolic heat-based AST study on Escherichia coli as model organism with 3 clinically relevant antibiotics is performed and the minimum inhibitory concentrations (MIC) are determined.
\end{abstract}

\section{Introduction}

Steadily increasing antimicrobial resistance (AMR) of medically relevant microorganisms is an important threat to global health 1-3. Multidrug resistance of common pathogens, such as Staphylococcus aureus, Enterobacteriaceae and Pseudomonas aeruginosa, brings about severe therapeutic challenges, associated with elevated morbidity and mortality rates ${ }^{4}$. An often cited AMR review report, based on detailed scenario analyses of the impact of AMR spreading on health-care and macroeconomics, estimated to 10 million the number of deaths per year attributable to AMR in 2050 , i.e. more than for most of the other major causes of disease-related death ${ }^{5}$. An European cross-national database study established a correlation between $\mathrm{AMR}$ and outpatient antibiotic use ${ }^{6}$. Moreover, clinical studies revealed a high percentage of unnecessary prescriptions of antibiotics for therapeutic and prophylactic purposes. This fact has been recognized as one of the main causes of emerging AMR. In many cases prescribed drugs are broad-spectrum antimicrobial agents, which further promotes AMR development and spreading.

Rapid diagnosis of bacterial infection combined with fast and accurate antimicrobial susceptibility testing (AST) is one of the keys to counteract AMR progression, in particular by optimizing and personalizing the therapeutic management of infected patients ${ }^{7}$. AST in vitro techniques aim to predict the efficiency

\footnotetext{
a. Laboratory of Microsystems, Ecole Polytechnique Fédérale de Lausanne, CH-1015 Lausanne, Switzerland.

Electronic Supplementary Information (ESI) available: [details of any supplementary information available should be included here]. See DOI: 10.1039/x0xx00000x
}

of an antimicrobial compound for treating an infection. Ideally, an AST protocol should provide the minimum inhibitory concentration (MIC) for microbial growth, i.e. the lowest drug concentration that inhibits bacterial growth after overnight incubation ${ }^{8}$. Standard AST methods in clinical microbiological diagnosis, in particular the disk diffusion method and broth microdilution (BMD), are based on established, robust and often manual protocols. A commercial diffusion-based gradient method (Etest ${ }^{\circledR}$, Biomérieux) may be used for MIC determination ${ }^{9}$. Current clinical protocols, however, suffer from important limitations, in particular extended time to result or the requirement for relatively large amounts of viable microorganisms. Automated AST systems based on turbidity measurements aim to reduce time to result, typically to below $24 \mathrm{~h}^{10}$. Moreover, a range of new AST technologies is under development to tackle the AMR problem ${ }^{10,11}$, either in a 96-well plate or microfluidic format, including molecular diagnostic tools to detect the presence of resistance genes ${ }^{12}$ and fast AST assays based on single-cell imaging, for instance ${ }^{13}$. Nevertheless, most of these technologies still face major barriers with respect to general acceptance by the health care system and market penetration ${ }^{7}$.

In this context, we explore microbial heat production as a direct indicator of metabolic activity and viability of bacterial populations. Metabolic heat/heat flow curves provide real-time information on microbial growth dynamics and can be used for detecting bacterial growth inhibition in the presence of antimicrobials in vitro. The potential of isothermal microcalorimetry (IMC) for microbial activity monitoring, metabolic studies and drug assays has already been demonstrated by means of commercial devices. The latter use 
sealed $\mathrm{mL}$-size sample ampoules and have very low heat power detection limits, typically in the range of a few $\mu \mathrm{W}$ down to 0.2 $\mu \mathrm{W}^{14,15}$. For instance, different metabolic phases of Escherichia coli (E. coli) bacterial growth have been identified by analyzing heat flow profiles ${ }^{16}$. Highly sensitive and fast detection of bacterial contamination ( $\geq 1-10 \mathrm{CFU} / \mathrm{mL}$ ) was also demonstrated by recording heat flow of artificially contaminated blood platelets samples ${ }^{17}$. The potential of IMC for rapid differentiation between methicillin-resistant $S$. aureus (MRSA) and methicillin-susceptible S. aureus (MSSA) was evaluated by von Ah et al. by using the antibiotics cefoxitin and oxacillin ${ }^{18}$. Subsequently, Baldoni et al. reported a study using a 48-channel batch calorimeter for the detection of methicillinresistance in $S$. aureus including genetically distinct clinical isolates ${ }^{19}$. MRSA isolates were correctly identified after $5 \mathrm{~h}$ by testing against cefoxitin, however, very high bacterial inocula (McFarland turbidity of 5) were used to reduce time to result. Yang et al. analyzed the growth rate of $E$. coli and the time to maximum heat power as a function of concentration for two different cephalosporins ${ }^{20}$. The two antibiotics tested affected the heat power curves in a clearly different manner. A more extensive AST study was performed by von Ah et al., analyzing heat flow curves for reference strains of $E$. coli and $S$. aureus for 12 different antibiotics. The results have been grouped by mode of antimicrobial action, revealing different thermal signatures for each compound family ${ }^{21}$.

Different microfabricated and microfluidic integration formats have been proposed for high-sensitivity nanocalorimeters. A miniaturized chip-based calorimeter, similar to the one presented in the present work, has been developed earlier by Higuera-Guisset et al.. The authors studied the effect of two different growth media and of the culture temperature on the metabolic heat curves of $E$. coli ${ }^{22}$. Another group used a flowthrough chamber chip module, enclosed by two high-precision thermostats, for the study of biofilm inactivation by predatory bacteria and for monitoring biofilm eradication with antibiotics 23-25. Johannessen et al. implemented a sub-nL open-reservoir assay for heat measurements on a small number of isolated living cells ${ }^{26}$. Torres et al. reported the microfabrication of 96 detector enthalpy arrays on polyimide membranes and implemented rapid electrostatic merging/mixing of droplets 27 The technology enabled studying kinetic parameters of enzymecatalyzed reactions ${ }^{28}$. Lee et al. proposed a more complex closed-chamber microfluidic approach with precise $\mathrm{nL}$ sample manipulation capabilities for measuring the heat of reaction of urea hydrolysis, for instance ${ }^{29}$. Inomata et al. developed a picocalorimeter for detection of heat produced by a brown fat cell attached to a Si microstage connected to a cantilevered $\mathrm{Si}$ sensor. Heat is sensed by the resulting shift in the resonant frequency ${ }^{30}$. An ultrasensitive micro-DSC (differential scanning calorimeter) for liquid protein sample characterization was demonstrated by Wang et al.. The device is based on vanadium oxide thermistors and a microfluidic dual-chamber calorimeter design ${ }^{31}$. Kim et al. recently reported a thin-film parylene microfluidic calorimeter with on-chip vacuum insulation. Measurement of cellular metabolic power changes upon controlled stimuli was demonstrated ${ }^{32}$. A more recent commercial microcalorimetry instrument (Symcel $A B^{\circledR}$ ) uses a well-plate format for tracking the activity of living cells and biological processes ${ }^{33}$.

In this work, we present an isothermal nanocalorimetry platform designed for monitoring microbial growth dynamics with high sensitivity, thus enabling fast AST based on metabolic heat measurements. The INCFAST system (acronym for "isothermal nanocalorimetry platform for fast AST"), which is operated in a microincubator format, was optimized with respect to high thermal stability, a prerequisite for fast detection of bacterial infection or establishing accurate antibiograms. We evaluated microbial heat generation under different culture conditions. More importantly, we performed a proof-of-concept study comprising metabolic heat AST assays of E. coli exposed to 3 commonly used antimicrobial drugs (ciprofloxacin, ampicillin and gentamicin). Specific features of the metabolic heat/heat flow curves showed clear variations as a function of the antimicrobial concentration and MIC values could be determined for the 3 compounds. Furthermore, in a modified version of the isothermal platform, an oxygen sensor was implemented, enabling heat and oxygen consumption measurements for identical culture conditions in parallel. This offered a more detailed phenotypic fingerprint of the microbial metabolic activity upon drug exposure, while optical density measurements over time of the bacterial cultures provided additional insight in the development of bacterial biomass.

\section{Results and Discussion}

\section{Nanocalorimetry platform and oxygen consumption measurements}

The nanocalorimetry platform is constructed around a sensing unit comprising a microincubator for bacterial culture and a thermopile sensor chip that detects heat flow corresponding to the metabolic heat power generated by the microbial sample during incubation. A dual chip arrangement allows increasing assay throughput or differential/control measurements. Several important modifications have been implemented with respect to a previous version of the system ${ }^{34}$, mainly aiming at improving specific requirements for allowing high-sensitivity detection and accurate recording of metabolic heat profiles. These features include high thermal stability and fluidic integration suitable for fast thermalization of the sample and drug solutions. Fig. 1a shows an exploded 3D view of the INCFAST platform. Sensor chip and microincubator are inserted in the central part of the $\mathrm{Al} / \mathrm{Cu}$ thermostat. A proportionalintegral-derivative (PID) temperature control system with several heating pads provides uniform temperature distribution and stabilization at the sample location within $\pm 250 \mu \mathrm{K}$ at $37^{\circ} \mathrm{C}$ (Fig. S1, mK-range for the previous version of the platform ${ }^{34}$ ). Consequently, a limit of detection (LOD) for heat power of microbial suspensions as low as $750 \mathrm{nW}$ was achieved (Fig. S2). The core of the platform comprises the dual chip system with microincubators, fluidic connections and electrical read-out for 
a

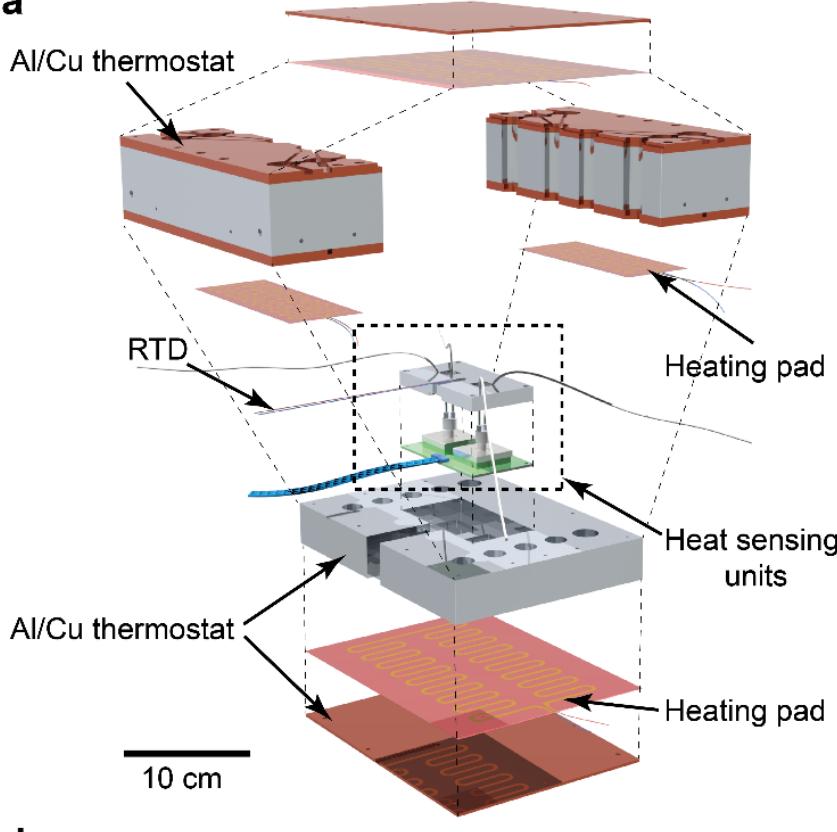

d

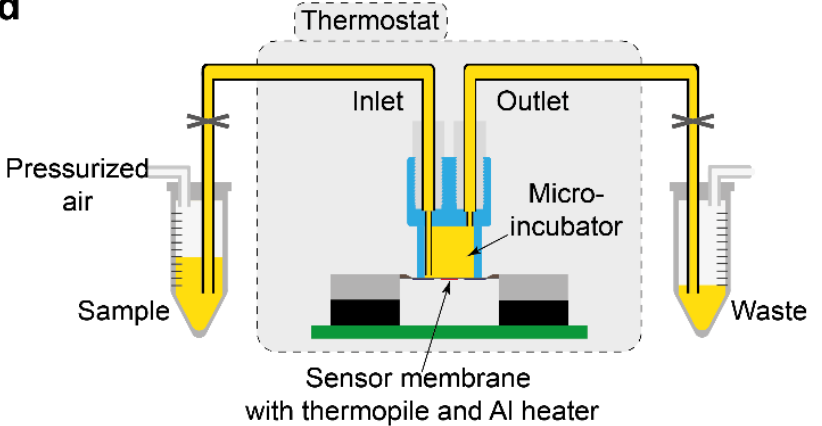

b

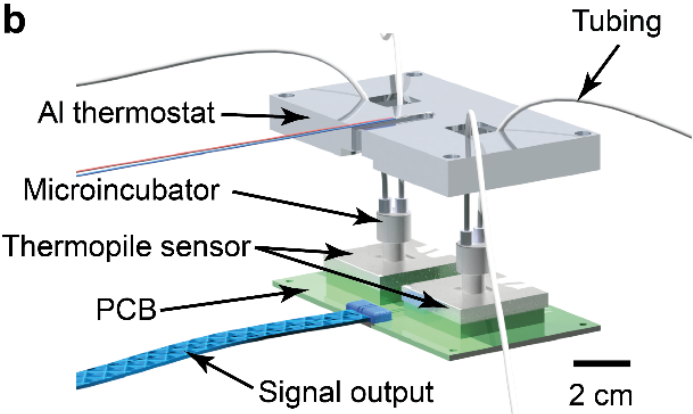

C

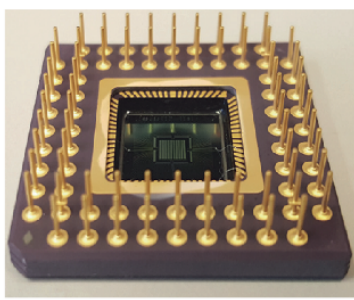

Thermopile sensor chip

e

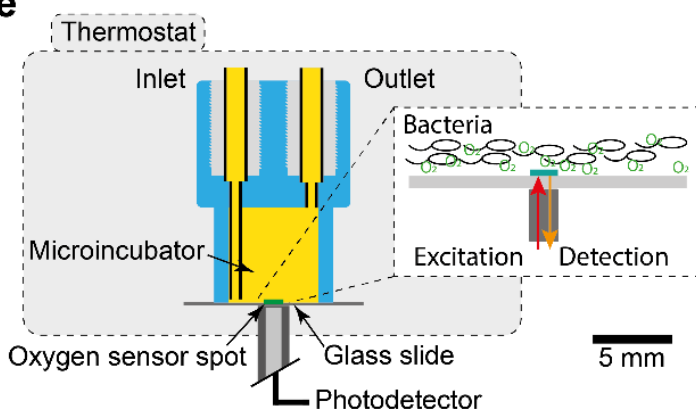

Fig. 1 The isothermal nanocalorimetry platform. (a) Exploded 3D view of the integrated platform with two thermopile-based sensing units. The Al/Cu thermostat is based on a proportional-integral-derivative (PID) controller, using several heating pads and a Resistance Temperature Detector (RTD) sensor (Pt1000). (b) Enlarged view of the twin heat sensing unit including a parallel arrangement of two microincubators $(2 \times 150 \mu \mathrm{L}$ ). (c) Photograph of one thermopile sensor chip and pin grid array (Xensor Integration, XEN-NCM9924). The hot junctions of the thermopile and a thin-film Al heating element are located on the sensitive central part ( $3 \mathrm{~mm} \times 3 \mathrm{~mm}$ ) of the $\mathrm{Si}$ membrane. The cold junctions are in thermal contact with the Si frame situated at the edge of the chip (10 $\mathrm{mm} \times 10 \mathrm{~mm}$ ). (d) Schematic cross-sectional view of a sensor unit with fluidic system (yellow). The microincubator is positioned directly on the chip membrane and connected to external sample and waste reservoirs, respectively. Bacterial suspensions are injected into the microincubator by pressure control of the sample reservoir. (e) Schematic cross-sectional view of the oxygen consumption measurement unit. The thin-film optical sensor spot (green, $\varnothing \approx 1 \mathrm{~mm}$ ), which shows oxygen-dependent luminescence in the near-infrared range, is integrated in a set-up similar to the nanocalorimetry platform. The oxygen concentration is measured after sedimentation of the bacterial population (see inset).

independent parallel measurements (Fig. 1b). Voltage signals generated by the thermal sensors are recorded by a twochannel nanovoltmeter (34420A, Keysight, USA). Commercially available sensor chips were used in this study (XEN NCM9924, Xensor Integration, Delft, The Netherlands) (Fig. 1c). The sensing mechanism is based on a thin-film thermopile distributed around the center region of a Si membrane. The relatively thick membrane ( $45 \mu \mathrm{m}$ according to the data sheet) provides sufficient robustness for liquid nanocalorimetry applications.

More details of the integrated system are shown in Fig. $1 \mathrm{~d}$. The microincubator that receives the bacterial suspension is bonded onto the sensor surface. The liquid sample is in direct contact with the Si sensor membrane (no polymer membrane on the bottom of the microincubator), thus maximizing heat transfer to the sensing elements. The microincubator has one sample inlet and one waste outlet. The corresponding reservoirs are located outside the thermostat at room temperature for convenient operation. Nevertheless, thermalization of compound solutions and bacterial suspensions during injection is fast and not limiting the performance of the platform for the present application involving relatively slow heat signal variations. Optionally, the reservoirs could be placed inside the thermostat for reducing thermal stabilization time. Placing the whole setup inside a foam/polystyrene housing (not shown) further reduced perturbations due to environmental temperature variations.

The INCFAST nanocalorimetry measurements were combined with real-time oxygen consumption measurements of a microbial suspension under culture conditions that are equivalent to the heat assays. For that purpose, the thermopile sensor was replaced by a glass substrate holding a sputter- 
a

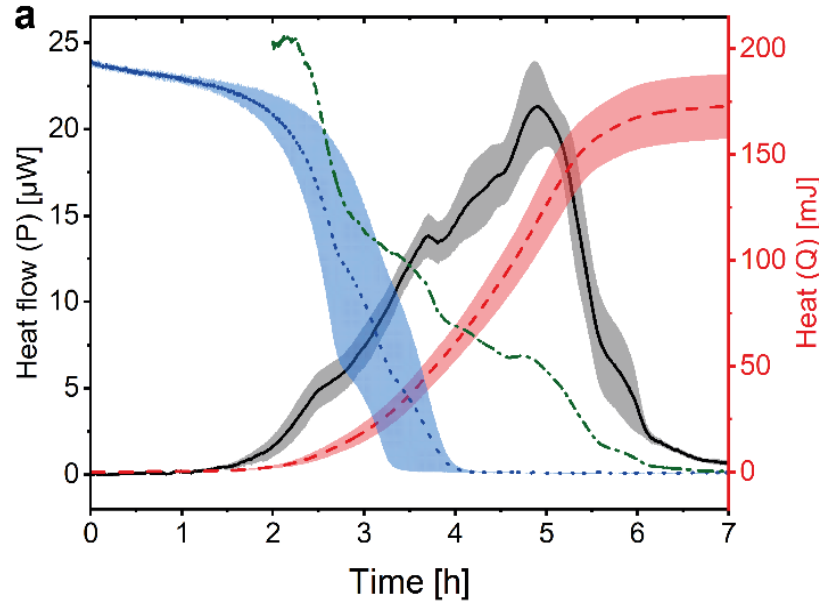

- Heat flow $\cdots$... Oxygen consumption

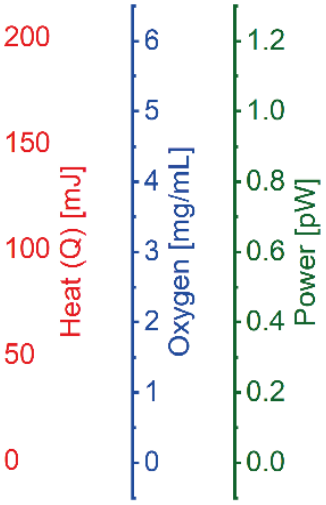

-.-. Single bacterial power

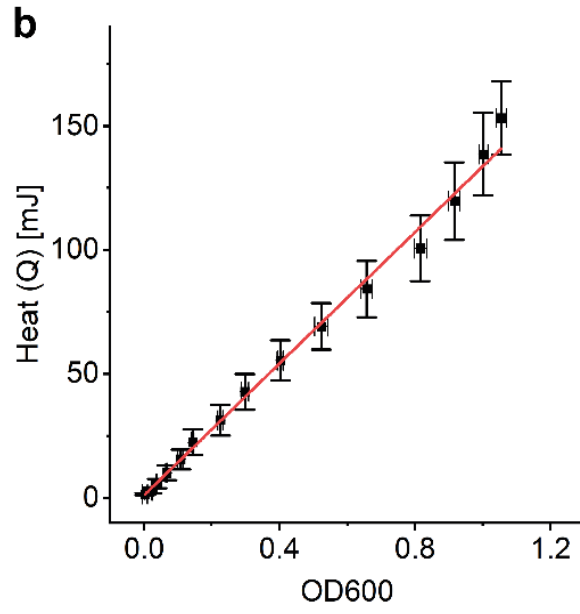

- Heat vs optical density Linear fitting

Fig. 2 Metabolic heat and oxygen consumption measurements performed on the nanocalorimetry platform. (a) Metabolic heat flow curve $P(t)$ (black) for $E$. coli ATCC 25922 with typical incubation conditions on the INCFAST platform (Mueller-Hinton broth (MH), $37^{\circ} \mathrm{C}$ ). The heat power signal was obtained by read-out of the thermopile sensor voltage output after calibration. Integration of the heat flow curve over time provides the corresponding metabolic heat curve $Q(t)$ (red). The single bacteria heat power (green curve) was estimated by normalization of $Q(t)$ by the biomass at a given time point, as determined from optical density (OD600) measurements. Synchronized parallel on-chip oxygen consumption measurements revealed complete oxygen depletion in the microincubator after $4 \mathrm{~h}$ (blue curve), indicating the transition from an aerobic to an anaerobic metabolic state. The assay interval for $t=[0,7 \mathrm{~h}]$ is emphasized in the plot. For $t>7 \mathrm{~h}$ heat values remain constant, all other values tend to zero. (b) Plot of the metabolic heat signal vs OD600 of a bacterial suspension up to the exponential growth phase (using data shown in (a) and Fig. S3a for $t=[0,5 \mathrm{~h}]$ ). The proportional relationship (coefficient $134 \mathrm{~mJ} / 1.0 \mathrm{OD} 600$ ) indicates that the heat signal is directly related to the amount of bacteria in this regime. All curves represent mean $\pm \operatorname{SE}(n=3)$.

deposited thin-film optical oxygen sensor spot (Fig. 1e). The sensor features detection of oxygen-dependent luminescence in the near-infrared (REDFLASH Technology, PyroScience $\mathrm{GmbH}$ ). The microincubator was fixed directly over the sensor and optical access was provided from the bottom of the thermostat. The same thermal control and fluidic systems were used for both platforms, ensuring identical assay conditions. Parallel heat and oxygen measurements were performed by injecting simultaneously identical bacterial samples into each platform.

\section{Analysis of microbial metabolic heat profiles}

Fig. 2a (black curve) shows a representative metabolic heat flow curve (i.e. heat power $P(t)[\mathrm{W}]$ ) for $E$. coli in Mueller-Hinton broth $(\mathrm{MH})$ recorded on the INCfAST platform $(V=150 \mu \mathrm{L}$, inoculum size $\left.2.8 \pm 0.5 \times 10^{5} \mathrm{CFU} / \mathrm{mL}, 37^{\circ} \mathrm{C}\right)$. The $P(t)$ curve displays the time-dependent metabolic heat flow generated by the bacterial population in the center region of the Si chip membrane towards the Si bulk edge, thus depends on the actual number of viable cells and their metabolic activity. In the initial segment the signal is below LOD, corresponding to an apparent lag phase or delay time $\left(t_{\text {delay }} \approx 1.5 \mathrm{~h}\right.$ ) associated with low biomass and/or low metabolic activity. Subsequently, exponential growth generates a rapidly rising signal up to a transient maximum rate of heat production $P_{\max }$ (at $\sim 5 \mathrm{~h}, 21.3 \pm$ $2.5 \mu \mathrm{W}$ ) followed by a sharp decline. Assay curves have been recorded up to $20 \mathrm{~h}$, however only the most relevant time interval $[0,7 \mathrm{~h}]$ is shown in Fig. 2a. At longer incubation times ( $\geq 10-11 \mathrm{~h}$ ) metabolic heat production ceased due to nutrient and/or oxygen depletion in the microincubator. At this stage, most of the bacteria are still alive as revealed by optical microscopy inspection or are possibly in a dormant state. The aggregate metabolic heat curve (energy $Q(t)[\mathrm{J}]$ ) was obtained by integration of the heat flow curve over time (Fig. 2a, red curve). Accordingly, the $Q(t)$ curve features three phases, namely the initial lag phase, the exponential growth region (for $t \sim[1.5 \mathrm{~h} ; 5.5 \mathrm{~h}]$ ) and a stationary phase (for $t>\sim 7 \mathrm{~h}$ ), in which the total heat does not increase any more $\left(Q_{\max } \approx 175 \pm 15 \mathrm{~mJ}\right)$. For the metabolic assays discussed below, the $P(t)$ and $Q(t)$ curves shown in Fig. 2a were used as reference for normal culture conditions on the INCFAST platform.

We performed optical density measurements (OD600, i.e. at $600 \mathrm{~nm}$ ) under culture conditions comparable to the BMD method on a plate reader. As shown in Fig. S3b for culture in $\mathrm{MH}$, the exponential phases of the $Q(t)$ and the OD600 curves overlap well. A scaling or heat/OD600 conversion coefficient of $134 \mathrm{~mJ} / 1.0 \mathrm{OD} 600$ for $\mathrm{MH}$ was determined by linear regression of the $Q(t)$ vs OD600 plot shown in Fig. 2b. This observation indicates that heat curves recorded with our system can be safely correlated with bacterial growth or the actual biomass density in the microincubator, respectively. Mean slopes $Q / t$ in the exponential region may be considered as indicative for bacterial growth rates, whereas heat flow profiles $P(t)$ are more sensitive to transient phenomena of the growth behavior. Furthermore, $P(t)$ can be normalized with respect to biomass simply by dividing through the converted $Q(t)$. The resulting curve (Fig. 2a, green curve), showing a continuous decline $(\sim 0$ $\mathrm{pW}$ for $t>7 \mathrm{~h}$ ), provides a rough estimate of the time evolution of the metabolic heat production per bacterium for the specific culture conditions. For the calculation we applied a conversion equivalence of $1.00 D 600$ corresponding to $5.0 \times 10^{8} \mathrm{CFU} / \mathrm{mL}^{35}$. 
In order to further analyze the metabolic phenotype, we measured the oxygen concentration during bacterial growth in the thermalized microincubator by modifying the nanocalorimetry platform into a luminescence detection-based oxygen sensor platform, taking care to keep culture conditions identical to those of the heat flow assay. For aerobic bacteria such as $E$. coli, oxygen consumption is an important indicator of metabolic activity and viability. The microincubator itself can be considered as gas non-permeable, thus the oxygen concentration directly reflects the actual oxygen consumption of the bacterial population. In the case study discussed in Fig. 2, the oxygen signal started declining sharply at the onset of exponential growth (i.e. at $\sim 1.5 \mathrm{~h}$ ) and reached a nondetectable level after $4 \mathrm{~h}$ (Fig. 2a, blue curve). Interestingly, the oxygen consumption curve indicates that depletion of oxygen occurs earlier than the time point were heat flow is maximum $P_{\text {max }}$ (at $\sim 5 \mathrm{~h}$, black curve in Fig. $2 \mathrm{a}$ ), suggesting that the bacteria population continues growing normally for at least $\sim 1 \mathrm{~h}$ in an anaerobic metabolic state. As a facultative anaerobic bacterium, E. coli thus appears not to be immediately sensitive to oxygen depletion. Indicative heat power/bacterium mid-range values are $1.1 \mathrm{pW}$ (at $t=2.5 \mathrm{~h}$ ) for the aerobic metabolic state and 0.32 $\mathrm{pW}$ (at $t=5 \mathrm{~h}$ ) for the anaerobic state, respectively (Fig. 2a, green curve). Transitions between different metabolic states may in principle result in more complex heat flow curves (see Discussion). In the present case, a small crinkle-like feature can be identified at $\sim 3.7 \mathrm{~h}$ (Fig. 2a, black curve), possibly corresponding to the transition from aerobic to anaerobic conditions. Nevertheless, no significant impact on the overall shape of the heat flow curve is observed, suggesting a fast shift between the two metabolic states.

\section{Metabolic activity of $E$. coli for different culture conditions}

Assessment of the metabolic activity of $E$. coli in different culture media (Fig. 3a, 3b and 3c), as well as for several culture temperatures (Fig. 3d, 3e and 3f), was performed as a first evaluation of the INCFAST platform performance. Heat flow curves have been recorded in Brain Heart Infusion medium (BHI), $\mathrm{MH}$ and Lysogeny broth (LB) (Fig. 3a). These media have different compositions and amounts of nutrients (Table S1), affecting growth rate and metabolic activity of the bacteria. We found that rich media, in particular $\mathrm{BHI}$, promotes transient metabolic heat production reflected by the $P_{\max }$ values of the heat flow curves, yielding $31.3 \pm 1.2 \mu \mathrm{W}$ in $\mathrm{BHI}$ (highest amount of carbon compounds), $21.3 \pm 2.5 \mu \mathrm{W}$ in $\mathrm{MH}$ (intermediate nutrient level) and $18.7 \pm 1.4 \mu \mathrm{W}$ in LB (low amount of nutrients) (Fig. 3a). Consequently, culture in $\mathrm{BHI}$ also enhanced the total heat generated during incubation ( $Q_{\max } \approx 252 \pm 10 \mathrm{~mJ}$ in Fig. $\left.3 \mathrm{~b}\right)$,

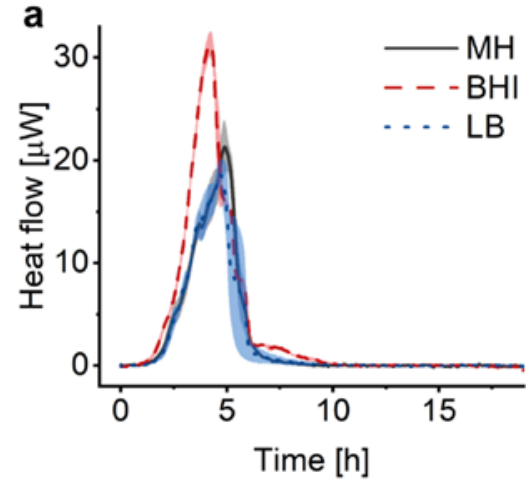

d

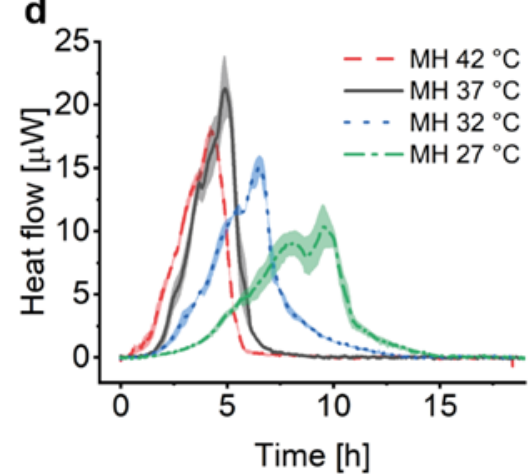

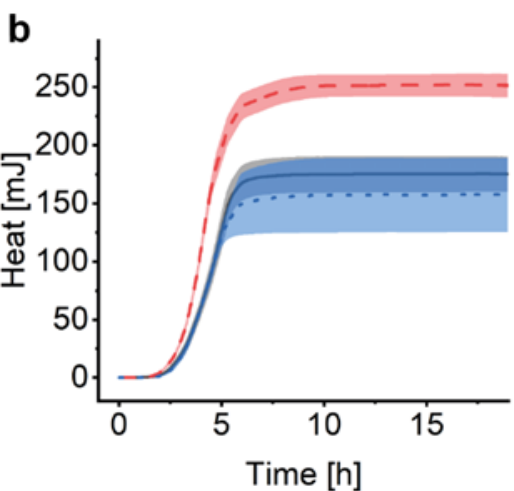

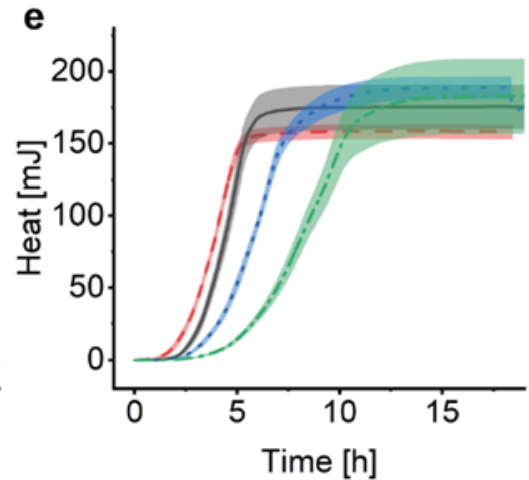

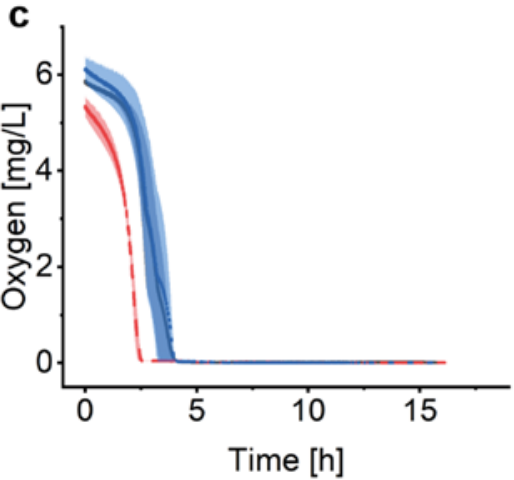

f

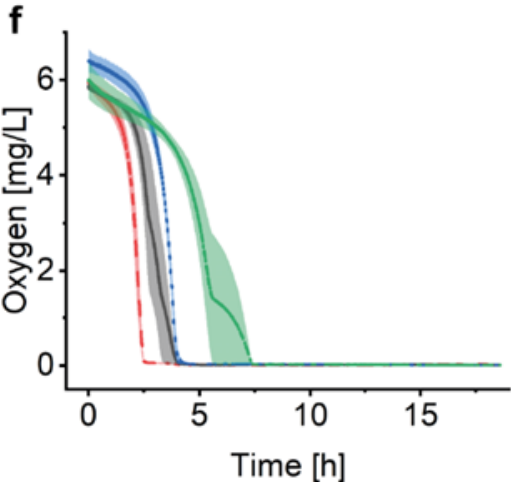

Fig. 3 Assessment of metabolic features for different growth conditions of E. coli ATCC 25922. (a) Metabolic heat flow $P(t)$ for different culture media at $37^{\circ} \mathrm{C}$. Rich medium (Brain Heart Infusion medium (BHI), black curve) enhances the maximum bacterial heat production rate compared to media containing less nutrients (Mueller-Hinton broth ( $\mathrm{MH}$ ), red curve, or Lysogeny broth (LB), blue curve). (b) Corresponding heat curves $Q(t)$ obtained by time integration of the curves in (a). The maximum total heat value increases with the amount of nutrients provided by the medium (BHI $>\mathrm{MH} \geq \mathrm{LB}$ ). (c) Bacterial oxygen consumption in different culture media. Oxygen depletion occurs first in the richest medium (BHI), whereas oxygen consumption rates in $\mathrm{MH}$ and LB are very similar. (d) Heat flow curves for different culture temperatures in $\mathrm{MH}$. The maximum peak value for heat flow decreases in the lower temperature range and secondary peaks are more pronounced. The apparent lag phase elongates significantly at $27^{\circ} \mathrm{C}$ (green curve). (e) Heat curves obtained by integrating the curves in (d), indicating comparable stationary phase end levels $Q_{\text {max }}$, but reached at different time scales. (f) Oxygen consumption of the bacterial populations at different temperatures. Oxygen depletion is fastest at $42{ }^{\circ} \mathrm{C}$ and more delayed for decreasing temperatures. All curves represent mean \pm SE $(n=3)$. 
whereas $Q(t)$ reached comparable levels for $\mathrm{MH}$ and $\mathrm{LB}$ in the stationary phase (175 $\pm 15 \mathrm{~mJ}$ and $157 \pm 32 \mathrm{~mJ}$, respectively) Oxygen depletion occurs earliest for $\mathrm{BHI}$, indicating fastest growth and/or highest metabolic activity, whereas oxygen curves are comparable for MH and LB (Fig. 3c).

For all media, the OD600 curves, recorded separately on a plate reader, overlap well with the corresponding heat curves in the exponential region (after $y$-axis scaling and time synchronization, Fig. S3a-c). OD600 measurements reveal that the increase of the bacterial biomass is fastest in $\mathrm{BHI}$ compared to $\mathrm{MH}$ or $\mathrm{LB}$. As already shown in Fig. $2 \mathrm{~b}$ for $\mathrm{MH}$, heat/OD600 coefficients were determined by linear regression in the exponential region for all media tested (Fig. S3d). These coefficients vary for the different culture media (BHI $84 \mathrm{~mJ} / 1.0$ OD600, MH $134 \mathrm{~mJ} / 1.0$ OD600 and LB $162 \mathrm{~mJ} / 1.0$ OD600), indicating that heat curves cannot be converted to growth curves based on biomass in a universal manner for all conditions. Considering the $P_{\max }$ values stated above, maximum growth rates of $1.9 \times 10^{5} \mathrm{CFU} / \mathrm{s}$ for $\mathrm{BHI}, 8.1 \times 10^{4} \mathrm{CFU} / \mathrm{s}$ for $\mathrm{MH}$ and 6.0 $\times 10^{4} \mathrm{CFU} / \mathrm{s}$ for $\mathrm{LB}$, respectively, can be derived for culture in the microincubator of the INCFAST platform $\left(1.0\right.$ OD600 $=5.0 \times 10^{8}$ $\mathrm{CFU} / \mathrm{mL}$ ). Incubation of $E$. coli in $\mathrm{BHI}$ yields the fastest growth rate but the lowest heat production rate. This finding is in agreement with a previous statement in literature, claiming that the heat production rate per unit weight is inversely proportional to the growth rate in the exponential phase ${ }^{36}$. Interestingly, for all 3 media conditions in the microincubator $Q_{\max }$ values are reached on the same time scale (at $t \approx 6 \mathrm{~h}$, Fig. $3 b)$.

We also compared the effect of culture temperature in $\mathrm{MH}$ on the heat flow curves (Fig. 3d) and the heat curves (Fig. 3e). As expected, bacterial populations show the highest transient growth rate (heat production rate) under most favorable conditions $\left(37^{\circ} \mathrm{C}, P_{\max } \approx 21.3 \pm 2.5 \mu \mathrm{W}\right)$ and lower values for higher $\left(42^{\circ} \mathrm{C}\right.$ ) or lower temperatures (in particular for $27^{\circ} \mathrm{C}$ with $\left.P_{\text {max }} \approx 10.3 \pm 1.8 \mu \mathrm{W}\right)$. The apparent lag phase $t_{\text {delay }}(\sim 1.5 \mathrm{~h}$ at $\left.37{ }^{\circ} \mathrm{C}\right)$ is shortest for $42{ }^{\circ} \mathrm{C}\left(t_{\text {delay }}<1 \mathrm{~h}\right)$ and becomes more important for decreasing temperatures $\left(\sim 3 \mathrm{~h}\right.$ at $\left.27^{\circ} \mathrm{C}\right)$. Further alterations of the heat flow curves, comprising a general broadening or flattening of the profiles and emerging secondary transient peaks, is observed for culture at low temperatures (Fig. $3 \mathrm{~d}, 32{ }^{\circ} \mathrm{C}$ and $\left.27^{\circ} \mathrm{C}\right) . P(t)$ broadening also results in lower mean slopes $Q / t$ in the exponential phase (Fig. $3 e$, in particular at $27^{\circ} \mathrm{C}$ ). Heat signals reach comparable $Q_{\max }$ levels for all culture temperatures (160 - $190 \mathrm{~mJ}$ ), but on longer time scales for decreasing temperature. Corresponding OD curves evolve similarly (Fig. S4). The temperature effect on oxygen consumption is in line with the heat curves as well, showing a clear delay of oxygen depletion at most unfavorable growth conditions $\left(27^{\circ} \mathrm{C}\right)$ (Fig. $\left.3 f\right)$.

\section{Antimicrobial susceptibility testing based on metabolic heat} assays
The INCFAST platform was primarily designed for probing the efficacy of antimicrobial drugs and possibly for monitoring specific modes of action by means of metabolic heat assays. The presence or absence of a heat signal during a preset incubation period provides information on antimicrobial susceptibility or resistance of a bacterial strain. We performed AST on E. coli ATCC 25922 by analyzing the metabolic heat profiles for a selection of clinically relevant antibiotics and estimated MIC values. Heat flow curves $P(t)$ for ciprofloxacin, ampicillin and gentamicin are shown in Fig. $4 \mathrm{a}, 4 \mathrm{~d}$ and $4 \mathrm{~g}$ and the corresponding heat curves $Q(t)$ in Fig. $4 \mathrm{~b}, 4 \mathrm{e}$ and $4 \mathrm{~h}$, respectively. $P(t)$ and $Q(t)$ reference curves without antibiotic (0 $\mathrm{mg} / \mathrm{L}, \mathrm{MH}, 37^{\circ} \mathrm{C}$ ) are the same as for the previous assays (Fig. $2 \mathrm{a}$ and Fig. 3 ). Fig. $4 \mathrm{c}, 4 \mathrm{f}$ and $4 \mathrm{i}$ are the oxygen consumption curves recorded for these assays. In the low drug concentration range and for all 3 antimicrobial agents tested, heat flow curves approximately maintain a shape similar to culture in pure medium (Fig. 4a, 4d and 4g, black and red curves, respectively), indicating normal exponential bacterial growth in the microincubator. A decrease of the maximum heat power $P_{\max }$ values and the appearance of bump-like features may be noted. Stronger alterations occur with increasing drug concentrations, especially for values approaching MICs. Heat signals disappear at concentrations above MIC (Fig. 4a, 4d and 4g, purple curves).

For ciprofloxacin, for instance, heat flow curves are relatively confined for drug concentrations $\leqslant 0.004 \mathrm{mg} / \mathrm{L}$, whereas at concentrations $\geq 0.015 \mathrm{mg} / \mathrm{L}$ heat production is inhibited (Fig. 4a). In the intermediate concentration range, i.e. for $0.008 \mathrm{mg} / \mathrm{L}$ in this case, the heat flow curve flattens significantly and secondary transient structures become more apparent. On the corresponding heat curve the mean slope $Q / t$ in the exponential growth region decreases (Fig. $4 \mathrm{~b}$, green curve). The total heat signal disappears at $\geq 0.015 \mathrm{mg} / \mathrm{L}$ for the assay duration of $20 \mathrm{~h}$. Moreover, a clear prolongation of the apparent lag phase $t_{\text {delay }}$ from $\sim 1.5 \mathrm{~h}$ at $0 \mathrm{mg} / \mathrm{L}$ to $\sim 4 \mathrm{~h}$ at $0.008 \mathrm{mg} / \mathrm{L}$ occurs. Based on this series of experiments, we assume that the MIC value for ciprofloxacin falls into the concentration interval $0.008 \mathrm{mg} / \mathrm{L}<$ $\mathrm{MIC} \leq 0.015 \mathrm{mg} / \mathrm{L}$. Clinical reference values established by the European Committee on Antimicrobial Susceptibility Testing (EUCAST) are summarized in Table 1 for the antibiotics studied in this work ${ }^{37}$. EUCAST provides a MIC range of $0.004 \mathrm{mg} / \mathrm{L}$ $0.016 \mathrm{mg} / \mathrm{L}$ for ciprofloxacin, thus the INCFAST result overlaps well with the reference range. 
a

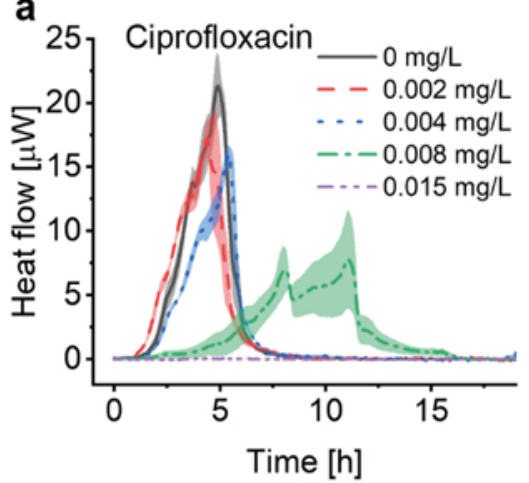

d
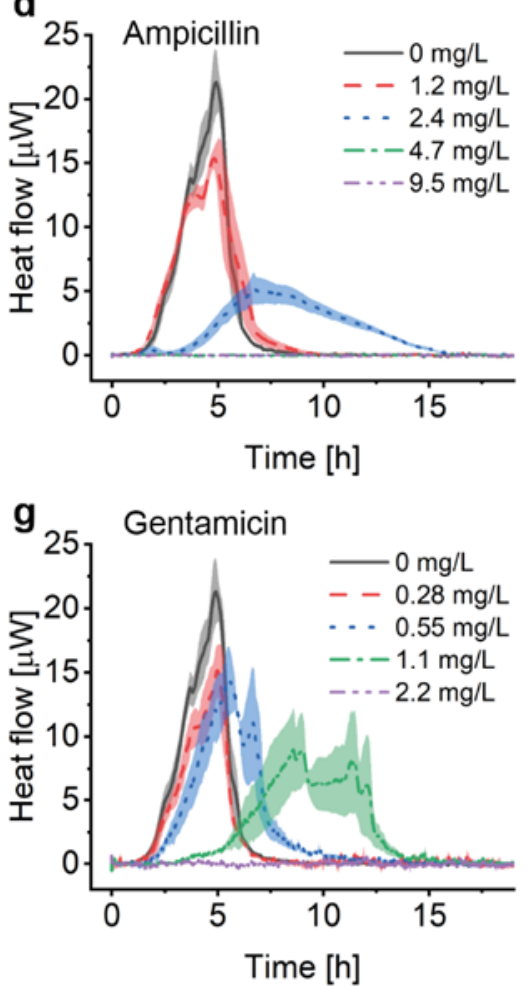

b

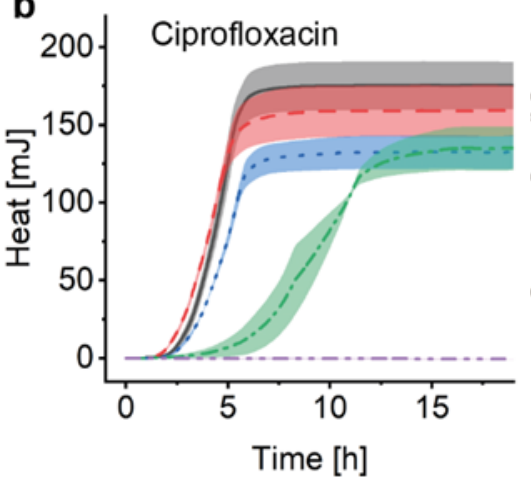

e

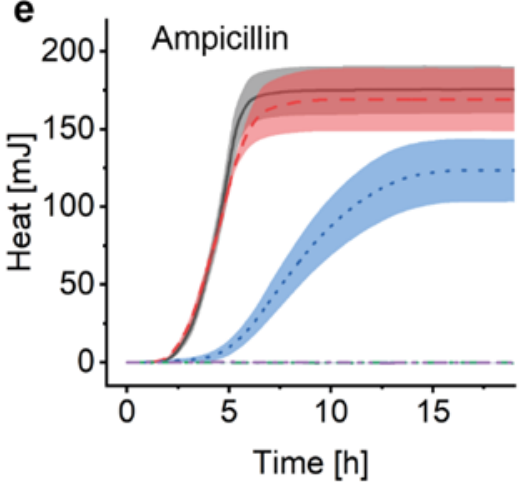

h

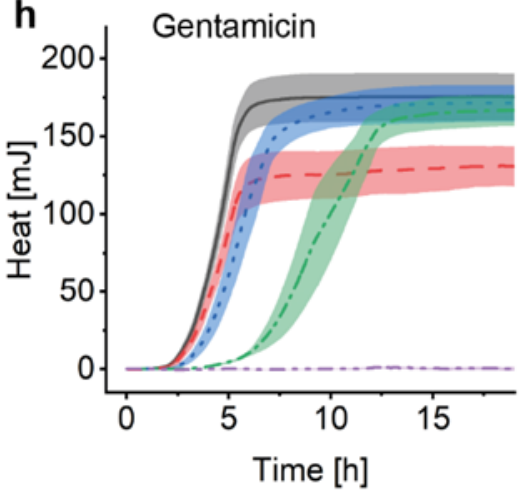

C

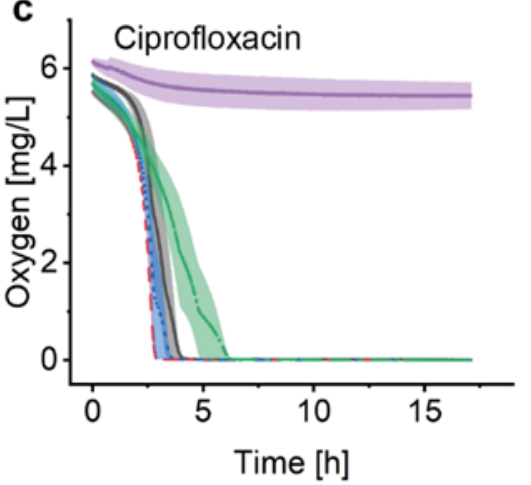

f
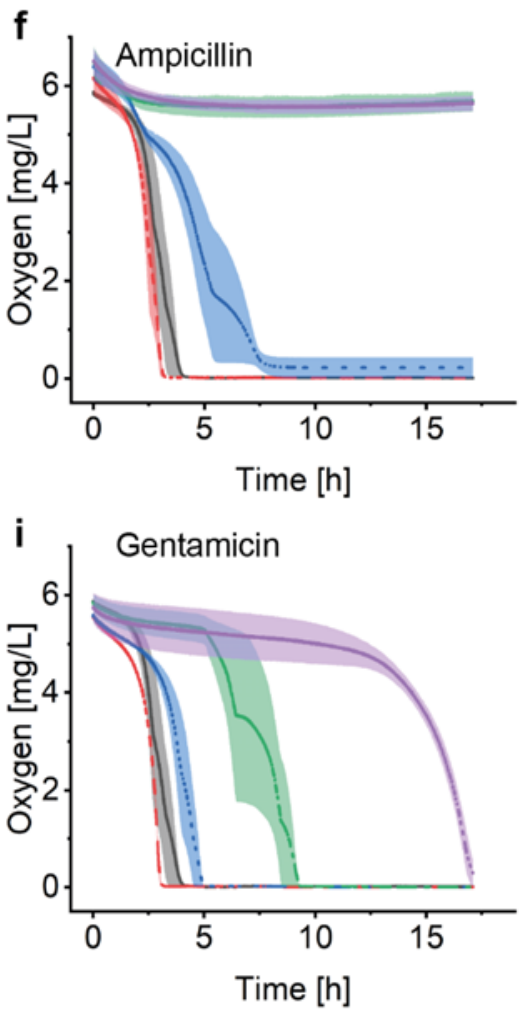

Fig. 4 Antimicrobial susceptibility testing with the nanocalorimetry platform. Heat flow curves for E. coli ATCC $25922\left(\mathrm{MH}, 37^{\circ} \mathrm{C}\right)$ recorded in the presence of different antibiotics and concentrations: (a) ciprofloxacin, (d) ampicillin, and (g) gentamicin. In all cases, a strong impact on the growth dynamics could be observed for antimicrobial concentrations approaching the minimum inhibitory concentrations (MICs). The MIC value for a specific antibiotic falls into the concentration interval, where complete suppression of the bacterial heat production occurs first. Corresponding heat curves are shown in (b), (e) and (h), respectively. In accordance to the heat flow curves, a general tendency of lag phase elongation is observed with increasing drug concentration. The absence of heat production above MIC is clearly observed. Furthermore, bacterial oxygen consumption curves (c), (f) and (i) have been recorded for the different pharmacological conditions. All 3 antimicrobials tested are bactericidal, thus the oxygen concentration in the microincubator should remain constant above MIC, as observed in (c) for ciprofloxacin (0.015 mg/L) and in (f) for ampicillin ( $4.7 \mathrm{mg} / \mathrm{L}$ and 9.5 $\mathrm{mg} / \mathrm{L}$ ). For gentamicin in (i), however, the oxygen concentration started dropping sharply after $\sim 13 \mathrm{~h}$ for $2.2 \mathrm{mg} / \mathrm{L}$, i.e. above MIC determined by heat flow, suggesting the presence of a small population of surviving bacteria near the oxygen sensor site. All curves represent mean $\pm \operatorname{SE}(n=3)$.

We performed similar AST assays for ampicillin (Fig. $4 \mathrm{~d}$ and Fig. 4e) and gentamicin (Fig. 4g and Fig. 4h). Ampicillin heat flow curves showed prolonged lag phase and a strongly modified profile at $2.4 \mathrm{mg} / \mathrm{L}$ (i.e. close to MIC). Secondary transient structures are not visible in this case. The mean slope $Q / t$ in the exponential growth region decreases strongly for this concentration (Fig. 4e, blue curve). Heat production was inhibited for higher concentrations ( $\geqslant 4.7 \mathrm{mg} / \mathrm{L}$ ) providing an INCfAST interval of $2.4 \mathrm{mg} / \mathrm{L}<\mathrm{MIC} \leq 4.7 \mathrm{mg} / \mathrm{L}$, which falls within the EUCAST reference value range $2 \mathrm{mg} / \mathrm{L}-8 \mathrm{mg} / \mathrm{L}$ (Table 1 ). Heat profiles for bacterial culture with increasing concentrations of gentamicin evolved in a similar way as for ciprofloxacin. Interestingly, close to MIC the secondary $P(t)$ transient features are strongly pronounced but the mean slope $Q / t$ in the exponential growth region seems to be less affected (Fig. $4 \mathrm{~g}$ and $4 \mathrm{~h}$, green curve). In case of gentamicin, however, the INCFAST interval $1.1 \mathrm{mg} / \mathrm{L}<\mathrm{MIC} \leq 2.2 \mathrm{mg} / \mathrm{L}$ was just above the EUCAST range (Table 1). As a control, we performed AST using the BMD method on a plate reader (Fig. S5). OD600 results for ciprofloxacin and ampicillin are consistent with heat measurements in terms of MIC values. The OD600 interval 0.55 $\mathrm{mg} / \mathrm{L}<\mathrm{MIC} \leq 1.1 \mathrm{mg} / \mathrm{L}$ for gentamicin is lower compared to the 
a

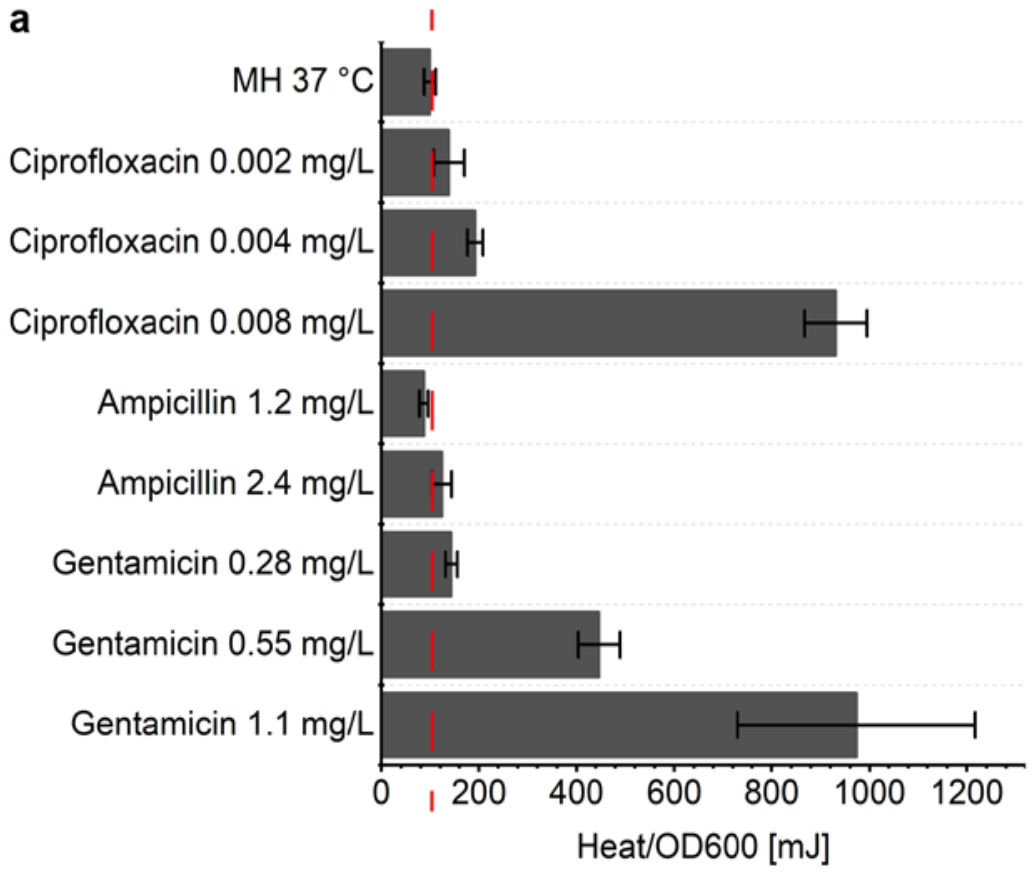

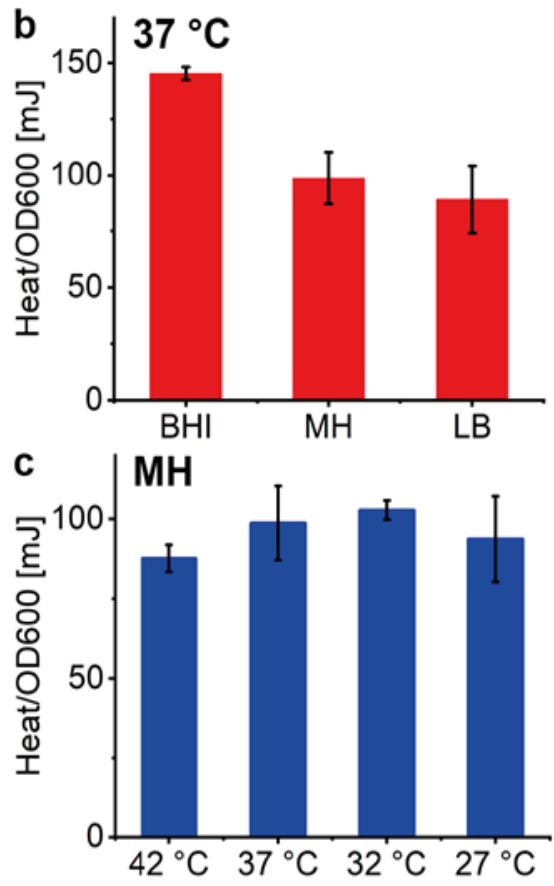

Fig. 5 Metabolic heat production vs optical density measurements of $E$. coli for different conditions. The plots show heat/OD600 coefficients, i.e. the ratio of total heat $Q_{\max }$ to the corresponding OD600 value. Both values were determined using the same sample at the endpoint of each assay after incubation on the INCfAST platform ( $t=$ $20 \mathrm{~h}$ ). (a) Coefficients for different antimicrobial exposures with respect to the reference value obtained for bacteria inoculated in the microincubator in pure $\mathrm{MH}$ ( 98 $\mathrm{mJ} / 1.0 \mathrm{OD} 600$, dashed line). The bar chart indicates that bacterial heat production per biomass unit is promoted by antimicrobial stress (except for ampicillin $1.2 \mathrm{mg} / \mathrm{L}$ ). (b) Heat/OD600 coefficients for different culture media yielding the highest value for the richest media (BHI) at the endpoint. (c) Bacterial culture at different temperatures did not significantly change the heat/OD600 coefficients. All bars show mean $\pm \mathrm{SE}(n=3)$.

heat assay and overlaps well with the EUCAST range of 0.25 $\mathrm{mg} / \mathrm{L}-1 \mathrm{mg} / \mathrm{L}$.

Table 1 Minimum inhibitory concentration obtained with the nanocalorimetry platform compared to standard EUCAST* values (E. coli ATCC 25922). Our metabolic heat experiments (INCFAST platform) allow defining intervals, into which actual MIC values fall. For ciprofloxacin and ampicillin these intervals overlap well with the MIC range established by EUCAST. For gentamicin, the resulting MIC interval is slightly higher, possibly due to reduced activity after oxygen depletion in the microincubator. (*The European Committee on Antimicrobial Susceptibility Testing)

\begin{tabular}{ccc}
\hline Antibiotic & $\begin{array}{c}\text { INCfAST } \\
\text { MIC interval }(\mathrm{mg} / \mathrm{L})\end{array}$ & $\begin{array}{c}\text { EUCAST } \\
\text { MIC range }(\mathrm{mg} / \mathrm{L})\end{array}$ \\
\hline Ciprofloxacin & $0.008<\mathrm{MIC} \leq 0.015$ & $0.004-0.016$ \\
\hline Ampicillin & $2.4<\mathrm{MIC} \leq 4.7$ & $2-8$ \\
\hline Gentamicin & $1.1<\mathrm{MIC} \leq 2.2$ & $0.25-1$ \\
\hline
\end{tabular}

Oxygen consumption as a viability indicator can be applied as another method to determine metabolism and MIC for nonanaerobic bacteria. For concentrations below MIC, oxygen is gradually consumed and the time to full oxygen depletion in the microincubator increases clearly for concentrations approaching MIC (Fig. 4c, 4f and 4i). For ciprofloxacin and ampicillin oxygen levels remain constant above MIC because of metabolic inactivity or death of the bacteria (purple curves in Fig. 4c and Fig. 4f). In the specific case of gentamicin, oxygen consumption at subinhibitory concentrations evolves in principle as for ciprofloxacin and ampicillin, even if delays are more pronounced close to the expected MIC (e.g. green curve in Fig. $4 \mathrm{f}, 1.1 \mathrm{mg} / \mathrm{L}$ ). Surprisingly, oxygen depletion is also observed at a concentration of $2.2 \mathrm{mg} / \mathrm{L}$, i.e. above the MIC value range derived from the heat assay. The oxygen concentration in the microincubator decreased sharply only after a prolonged incubation period of $\sim 13 \mathrm{~h}$ (purple curve in Fig. $4 \mathrm{i})$, indicating that a small population of bacteria was able to survive in the microincubator. The antimicrobial action of gentamicin is based on an oxygen-dependent process ${ }^{38}$. It is possible that diffusion of oxygen to bacteria clusters attached on the surface of the oxygen sensing spot is limited thus inhibiting temporarily the action of gentamicin.

\section{Energy spilling of $E$. coli due to antimicrobial stress}

OD600 growth curves recorded with the BMD method on a plate reader show that the overall biomass density decreases for increasing antimicrobial concentration in the observed assay duration for the 3 compounds tested (Fig. S5), whereas observing a systematic behavior of the heat signals $Q_{\max }$ is less obvious (Fig. 4b, 4e and 4h). This observation implies that the metabolic heat production per biomass unit may be enhanced under antimicrobial stress. In order to analyze the correlation of heat production and biomass in the presence of antimicrobials more accurately, we measured OD600 values of samples directly collected from the microincubator at the end of each metabolic heat assay (at $t=20 \mathrm{~h}$ ). By this means, we may evaluate precisely the $Q_{\max }$ to OD600 ratio of the final bacterial suspension, thus providing an average value resulting from the 
incubation process on the INCFAST platform. Fig. 5a summarizes the heat/OD600 coefficients for different antimicrobial exposures below MIC and compares these values to bacterial growth without antibiotics $(98 \pm 12 \mathrm{~mJ} / 1.0$ OD600 for $\mathrm{MH}$, dashed line). The plot indicates that for ciprofloxacin and gentamicin the bacterial heat production per biomass unit is strongly promoted by antimicrobial stress, reaching a more than 9-fold enhancement close to MICs (931 $\pm 64 \mathrm{~mJ} / 1.0$ OD600 at $0.008 \mathrm{mg} / \mathrm{L}$ for ciprofloxacin and $970 \pm 243 \mathrm{~mJ} / 1.0$ OD600 at $1.1 \mathrm{mg} / \mathrm{L}$ for gentamicin, respectively). The effect is much less pronounced for ampicillin. These values, taken at the end of the assay, are indicative for comparing the impact of the different antibiotics, but absolute heat/OD600 coefficients may be different if taken during the growth phase at an earlier time point than in the stationary phase, as the biomass may still evolve.

A similar analysis based on assay endpoint detection of OD600 and $Q_{\max }$ has been done for different culture media (Fig. $5 \mathrm{~b}$ ). In this case, the heat/OD600 coefficients decreased from $\mathrm{BHI}, \mathrm{MH}$ to $L B$, corresponding to the order of the respective nutrient levels (98 $\pm 12 \mathrm{~mJ} / 1.0$ OD600 for $\mathrm{MH}$ ). This was not the case with the coefficients derived previously by linear regression during the exponential growth phase (Fig. 2b and Fig. S3d, 134 $\pm 3 \mathrm{~mJ} / 1.0$ OD600 for $\mathrm{MH}$ ). OD600 values have been determined either with a sample retrieved from the closed microincubator (Fig. 5 b) or cultured by BMD on a shaking plate reader (Fig. $2 b$ and Fig. S3d). Both methods evaluate the heat/OD600 coefficients in a methodically different way, with focus on averaging over the assay duration or on the exponential growth region, respectively. As outlined above, the endpoint method is better suited for evaluating energy spilling over the whole assay duration. For the average heat per biomass at different culture temperatures in $\mathrm{MH}$, no significant differences were observed (Fig. 5c).

\section{Discussion}

Current AST protocols in clinical microbiological laboratories generally require several incubation cycles for preparing standardized inoculums of isolated microorganisms and/or for pathogen identification. Reliable detection of growth inhibition typically again takes up to $\sim 20 \mathrm{~h}$, or even longer depending on the bacterial species. As a consequence, AST results may be available to clinicians only after several days ${ }^{9,10}$. Hence, in order to improve antimicrobial prescription and treatment practice, there is a clear need for implementing new technologies enabling fast and fully automated AST protocols $7,10,11$.

IMC has high potential for biomedical applications in general and for microbial studies in particular ${ }^{14-16}$. In this view IMC may also be considered as one of the near-future alternatives for AST in clinical settings ${ }^{11}$. For instance, among other specific advantages, calorimetric assays can in principle be performed directly with opaque clinical samples, thus reducing significantly time to result. The non-specificity of the heat signal, however, requires carefully designed protocols in order to extract the information of interest and interpretation of calorimetric data in terms of meaningful microbiological parameters ${ }^{15}$. Despite their performance, commercial IMC systems have drawbacks related to the use of sealed $\mathrm{mL}$-size ampoules, requiring relatively large inoculum size and impeding fluidic manipulations during the assay. The chip-based nanocalorimetry platform developed in this work aims to overcome such bottlenecks, mainly through miniaturization and improved fluidic integration. The INCfAST platform was designed for direct injection of bacterial suspensions or drug solutions into the isothermal microincubator. This feature reduces delay times (only the small sample/drug volume needs to be thermalized), enables automated operation and eventually enhances assay versatility. Minimizing thermal time constants by reducing sample volumes and thermostat size, combined with high sensitivity, is a prerequisite for implementing fast AST protocols.

In the present proof-of-concept study, AST assays were performed on purified samples with volumes of only $150 \mu \mathrm{L}$. In order to estimate the lower limit of time to detection, we consider the LOD of $750 \mathrm{nW}$ of the platform, corresponding to $\sim 10^{6} \mathrm{CFU}$ (assuming $1.1 \mathrm{pW}$ per E. coli bacterium). For an inoculum size of $4.2 \times 10^{4} \mathrm{CFU}\left(2.8 \times 10^{5} \mathrm{CFU} / \mathrm{mL}\right)$ and taking a typical doubling time of $15-20 \mathrm{~min}$ for $E$. coli in laboratory conditions, the limit of quantification (i.e. $3 \times \mathrm{LOD} \approx 2.2 \mu \mathrm{W}$ or $\sim 2.2 \times 10^{6} \mathrm{CFU}$ per microincubator) should be reached after $\sim 2$ h. In our study, heat flow was indeed reliably detectable after a time lapse of $\sim 2 \mathrm{~h}$ (corresponding to $\sim 2 \mu \mathrm{W}$ ) under normal culture conditions $\left(\mathrm{MH}\right.$, no antibiotics, $37^{\circ} \mathrm{C}$ ) (Fig. 2a). An additional conservative time lapse of $\mathbf{3 0} \mathrm{min}$ for thermalization was allowed after the injection before starting data recording of each experimental curves. This estimation is based on a fastgrowing laboratory strain of $E$. coli as bacterial model.

Based on the heat flow curves in Fig. 4, AST on E. coli ATCC 25922 with ampicillin, ciprofloxacin and gentamicin could be safely performed in approximately 5-6 hours using the INCfAST platform. After this incubation duration, a significant difference between the heat flows of the lag phase with antibiotic and the "no growth" of cultures at the MIC occurs in all 3 cases. Nevertheless we have to consider that most clinical isolates, for instance Klebsiella pneumonia ${ }^{39}$, Acinetobacter baumannii ${ }^{40}$, and Pseudomonas aeruginosa ${ }^{41}$ have doubling times in the range of 40 to $140 \mathrm{~min}$, i.e. longer than the $E$. coli strain in the present study. Accordingly, the amount of microbials required for reliable detection might only be reached on a longer time scale. On the other hand, the heat power per bacterium also varies for different species. Higher heat power relieves partly the adverse effect of slow growth in terms of time to result. Further investigations will be needed to evaluate the performance of the present approach in real clinical settings and to determine actual realistic time scales of the metabolic heat AST assays.

Compared to other AST techniques, the thermal profiles of metabolic heat assays reveal significantly more information on the mode and kinetics of action of antimicrobial compounds. In 
the frame of this study, we observed clear variations of growthrelated patterns in the presence of antibiotics. One important parameter is the time lapse to reach a detectable heat signal $t_{\text {delay }}$ (apparent lag phase). Extension of $t_{\text {delay, }}$ in particular for concentrations close to MICs, was observed for the 3 compounds tested (Fig. 4). This feature may be interpreted as a transient bacteriostatic effect, especially in the case of ciprofloxacin and gentamicin, where the mean growth rate $Q / t$ in the delayed exponential phase is less affected than for ampicillin. On the other hand, ciprofloxacin, ampicillin and gentamicin are bactericidal antibiotics and in principle two reasons may be considered for longer apparent lag phases: (i) the drug compound kills most of the bacteria population, but a small amount of surviving cells (persisters) can be detected only with delay ${ }^{42}$, or (ii) elongation of the lag phase of the bacterial growth cycle attributed to enzymatic adaptations or genetic regulations in response to changing micro-environmental biochemical conditions 43,44 . Single-cell analysis would be necessary for discriminating between these two possibilities. It has been shown previously that lag phase extension in the presence of antibiotics is an important criterion that has to be taken into account, in addition to MIC or IC50 (concentration at $50 \%$ growth inhibition) values, in order to evaluate the efficacy of a drug ${ }^{45}$. For instance, bacterial strains were found to increase the lag time as a strategy for developing compound tolerance in response to antibiotic stress ${ }^{46}$. Such findings are important for the clinical evaluation of antimicrobial treatments. As outlined above, the high sensitivity of the INCFAST platform allows bacterial growth detection using a standard inoculum size in pure medium after only $\sim 2.5 \mathrm{~h}$ for $E$. coli. Detection of infection in clinical samples should in principle be possible on the same time scale. Lag phase extension in the presence of antibiotics, however, counteracts this advantage. Nevertheless, even if antimicrobial action delays the onset of metabolic heat production, heat signals could still be detected as early as $\sim 4 \mathrm{~h}$ just below MICs for the specific conditions of our assays (Fig. 4). However, actual time windows in clinical settings for differentiation between growth/growth inhibition based on standard methods are set to 16-20 h. For implementing a new AST approach this time frame has to be reconsidered in order to take full advantage of the new technology, but also for defining an acceptable minimum assay time with respect to reliability of the results from the clinician's point of view.

Antimicrobial action not only delays the onset of heat production, but also has a significant impact on the transient features of the heat flow curves. Progressively emerging secondary peaks and a general broadening of the curves with increasing antimicrobial concentration is most evident in Fig. 4 a and $4 \mathrm{~g}$ for ciprofloxacin and gentamicin, in particular close to MICs (green curves). Similar features were also observed in heat profiles without drug exposure and are generally attributed to metabolic transitions. Metabolizing sequentially two different carbon sources results in successive growth cycles with multiple exponential phases, possibly separated by a phase with reduced growth rate or transient growth inhibition, giving rise to diauxic shifts ${ }^{47,48}$. As observed in Fig. $3 d$, such effects may be enhanced at low culture temperatures, possibly due to reduced cellular enzymatic activity. Transitions between respiration and a fermentation states (Fig. 2a, black curve) may also generate more or less pronounced features or peaks, depending on the respective time scales ${ }^{49}$. It was noted previously that deconvolution of heat profiles yields individual Gaussians that can be used to estimate the metabolic heat produced by different mechanisms ${ }^{16}$.

Comprehensive understanding of the dynamic growth behavior at subinhibitory concentrations revealed by specific metabolic heat flow profiles might enable anticipation of antimicrobial efficacy and possibly extrapolation to MIC values, provided that quantification of growth parameters through adequate growth models can be applied ${ }^{21,50}$. Interestingly, it was demonstrated by von Ah et al., based on an IMC study with 12 antibiotics on $E$. coli and $S$. aureus that it could be possible to distinguish between different modes of antimicrobial action. In addition to alterations of transient features, variations in $t_{\text {delay }}$ and mean growth rates $Q / t$ were observed. The 3 antibiotics tested in our nanocalorimetric study have different modes of action. Ciprofloxacin targets topoisomerase II (DNA gyrase), which inhibits bacterial DNA synthesis, ampicillin inhibits cell wall synthesis and gentamicin inhibits bacterial protein synthesis through binding to the $A$ site of $16 \mathrm{~S}$ ribosomal RNA ${ }^{51}$. As discussed before, we observed a strong impact on the heat profiles for all compounds. Nevertheless, no conclusions can be drawn from our results with respect to different mode of actions. Heat flow curves for ciprofloxacin (Fig. 4a) and gentamicin (Fig. 4c) close to MIC are very similar, for ampicillin (Fig. 4b) the overall shape is different. A more systematic study is necessary for further going interpretations. Investigation of bactericidal and bacteriostatic modes of action would also be of interest.

Total heat curves $Q(t)$ as integrals of heat flow curves allow a better evaluation of mean growth rates $Q / t$ in the exponential region. The aggregated heat value $Q_{\max }$ in the stationary phase is determined by growth-limiting factors, such as oxygen/nutrient depletion and waste production in the microincubator. Interestingly, comparing heat curves (Fig. 4) and OD600 values of samples after incubation in the microincubator revealed that the metabolic heat production may be enhanced under antimicrobial stress (Fig. 5), an observation that can be understood as energy spilling. Inefficient use or spilling of adenosine triphosphate (ATP) as energy source is known as a possible survival strategy for many bacterial species. In E. coli, energy spilling could be mediated by a futile cycle of potassium or ammonium ions ${ }^{52}$. As far as can be deduced from our nanocalorimetric assays (Fig. 5a), ciprofloxacin and gentamicin induced strong energy spillage close to MICs, whereas the effect was only weakly pronounced for ampicillin. Previously, an initial increase of the heat production rate upon antibiotic exposure was observed in Pseudomonas putida biofilms and related to energy-dependent resistance mechanisms ${ }^{53}$. In addition, there is evidence that antibiotics can perturb bacterial respiration and central 
metabolism. A recent publication demonstrated that antibioticinduced adenine limitation increases purine biosynthesis and ATP demand, which might relate antimicrobial stress to enhanced bacterial heat production ${ }^{54}$.

Combining oxygen consumption measurements with metabolic heat recordings of bacteria under antimicrobial stress provides a more holistic phenotypic characterization of metabolic activity. Time to full oxygen depletion in the microincubator increases clearly close to MIC (Fig. 4c, $4 \mathrm{f}$ and $4 \mathrm{i}$ ). In our assays, oxygen depletion appeared on a shorter time scale than significant features of the heat flow profiles (e.g. earlier than the maximum of heat production $P_{\text {max }}$ ). Oxygen consumption may therefore be explored as another potential parameter for fast AST on aerobic bacteria. Nevertheless, oxygen depletion in the microincubator has to be considered carefully when evaluating the results. For instance, the MIC interval for gentamicin determined with the INCFAST platform was above EUCAST reference values (Table 1). The oxygen-dependent antimicrobial action of gentamicin may be at the origin of this observation ${ }^{38}$. Progressive oxygen depletion in the microincubator during the assay possibly reduced the antimicrobial efficacy of gentamicin, resulting in somewhat enhanced MIC values with respect to conventional protocols. Accordingly, for OD measurements (Fig. S5c), where shaking of the plate reader ensures oxygen replenishment of the culture solution, no deviation from the standard MIC range was observed. On the other hand, the enclosed microincubator system might be suitable for performing fast AST on anaerobic bacteria, which can be challenging with the conventional agarbased culture methods.

\section{Experimental}

\section{Microincubator fabrication}

Polycarbonate microincubators $(V=150 \mu \mathrm{L}, \varnothing=5.7 \mathrm{~mm}$, height $=6 \mathrm{~mm}$ ) fabricated by micromilling were employed for the metabolic heat assays on the INCfAST platform. Polycarbonate offers good mechanical stability and high chemical resistance with respect to the sterilization protocols applied for the experiments.

\section{Sensor calibration}

Sensor calibration was performed by means of the Al thin-film resistive heater on the sensing area of the chip membrane. A deionized (DI) water-filled microincubator was positioned on the sensor during calibration in order to simulate actual assay conditions. Calibration of the thermopile voltage output was performed by generating defined heat power signals via an external electrical power source. We determined a power/voltage coefficient of $0.88 \mathrm{~W} / \mathrm{V}$ for the present system configuration (Fig. S6). This value is about 3 times higher than in the previous version of the platform $(0.29 \mathrm{~W} / \mathrm{V}){ }^{34}$. This improvement was mainly achieved by optimizing the heat transfer from the sample in the microincubator to the sensor membrane.

\section{Microbial sample preparation}

Assays in this work were carried out using the E. coli ATCC 25922 strain (E. coli WDCM 00013 Vitroids $^{\mathrm{TM}}$, Sigma-Aldrich). This organism is a typical AST control strain. We used a standard BMD protocol for bacterial culture and sample preparation. $E$. coli inoculum was first introduced into liquid $\mathrm{MH}$ culture medium and incubated overnight at $37^{\circ} \mathrm{C}$. Subsequently, $10 \mu \mathrm{L}$ of this suspension was cultured on $\mathrm{MH}$ agar plates (Thermo Scientific, CM0337) and diluted by streaking. Single colonies were picked to prepare a bacterial suspension with an OD value of 0.2 measured at $600 \mathrm{~nm}$ ( 0.5 McFarland standard), i.e. a concentration of $\sim 1 \times 10^{8} \mathrm{CFU} / \mathrm{mL}$. By dilution in pure culture medium we obtained the primary samples for the heat flow assays with an initial bacterial concentration of $2.8 \pm 0.5 \times 10^{5}$ $\mathrm{CFU} / \mathrm{mL}$ for all experiments (confirmed by 3 serial quality control experiments). A purity, viability and concentration check prior to each measurement series was carried out by inoculation on a non-selective agar plate (after further 100-fold dilution) and colony counting after overnight culture. With our quality control experiments) we determined a concentration of 3.7-5.5 $\times 10^{8} \mathrm{CFU} / \mathrm{mL}$ for $1.0 \mathrm{OD} 600$, which is consistent with the value given by Stevenson et al. ${ }^{35}$. The bacterial suspension was stored in a small flacon and used within $15 \mathrm{~min}$ as inoculum on the INCFAST platform. For some of the metabolic heat assays in this work different culture media were compared, including BHI (Thermo Scientific, CM1135), MH (Thermo Scientific, CM0405), and LB (Sigma Aldrich, L3522). All culture media and agar plates were prepared according to the recommended protocols and concentrations. Microbial suspensions and antimicrobial solutions for OD600 measurements were prepared in the same way as for the heat assays. We performed OD600 measurements on a plate reader (24-well plate, inoculum $2.8 \pm$ $0.5 \times 10^{5} \mathrm{CFU} / \mathrm{mL}, 37^{\circ} \mathrm{C}$ ). The microbial suspension in each well was sealed with $500 \mu \mathrm{L}$ mineral oil (Sigma-Aldrich, M8410) to prevent evaporation.

\section{Antimicrobial solutions}

Ciprofloxacin, ampicillin sodium salt, and gentamicin were purchased from Sigma-Aldrich. Ampicillin sodium salt and gentamicin are soluble in DI water. $1 \mathrm{~mol} / \mathrm{L}$ hydrogen chloride solution was used for ciprofloxacin. Ciprofloxacin, ampicillin, and gentamicin stock solutions were prepared with a concentration of $25.5 \mathrm{mg} / \mathrm{mL}, 51.9 \mathrm{mg} / \mathrm{mL}$ and $36.0 \mathrm{mg} / \mathrm{mL}$, respectively. Stock solutions were stored at $4{ }^{\circ} \mathrm{C}$ and used within maximum 1 week to avoid a risk of declining activity. Prior to the measurements stock solutions were thermalized at room temperature and gradually diluted in DI water to prepare a series of concentrations according to the potency of each antibiotic (10 times higher than the final target concentrations). Test solution aliquots were prepared by further 10 -fold dilution in $\mathrm{MH}$. Target concentrations were chosen according to the range suggested by EUCAST. The bacterial inoculum was added 
to the test solutions immediately before sample injection into the microincubator.

\section{Platform sterilization}

In order to avoid damage of the fragile sensor membrane during operation, different key components, in particular the microincubator and the sensor, were generally reused in successive measurements. After each experiment, a cleaning and sterilization protocol was applied to the whole fluidic circuit of the nanocalorimetry platform, including tubing, the microincubator and the sensor surface. First, the circuit was thoroughly rinsed with ethanol in order to remove and elute most of the bacteria in the system, followed by soaking all exposed surfaces in RAPICIDE ${ }^{\mathrm{TM}}$ OPA/28 High-Level Disinfectant (Cantel Medical Corp.) for 20 min for sterilization and repeated washing with sterilized DI water. Control experiments without bacterial inoculum confirmed the efficiency of this protocol (no microbial heat signal was detected for $\leq 15 \mathrm{~h}$, Fig. S2). The platform and all auxiliary devices were kept under a biosafety cabinet to avoid contamination from the environment during the experiment.

\section{Heat flow measurement protocol}

Metabolic heat generation of E. coli ATCC 25922 was studied under different culture conditions, and upon exposure to antimicrobial compounds. First, the microincubator was installed in the platform and connected to the reservoirs for the bacterial sample/drug suspension and waste, respectively (Fig. 1d). The platform was then stabilized to the temperature set point (normally $37{ }^{\circ} \mathrm{C}$ ) and kept at that temperature for consecutive measurements. The sample solution was injected into the microincubator by pressure control of the sample reservoir (Fluigent MFCS pressure pump). The microincubator was then isolated by closing the valves in the inlet/outlet tubing and recording of the heat flow curves was started. Different assay conditions were successively tested by changing the external sample reservoir and refilling the microincubator after applying the cleaning/sterilization protocol described above. The platform thermalization was not interrupted. From the initial part of the experimental recordings, we found that thermal stabilization of the injected solution and the sensor can be safely achieved within $30 \mathrm{~min}$. On the heat flow curves $P(t)$ and heat curves $Q(t)$ shown in this paper, $t=0 \mathrm{~h}$ defines the time point when signal recording was actually started, i.e. $30 \mathrm{~min}$ after solution injection. Heat flow curves were generally recorded through the whole culture cycle (sampling frequency: $0.5 \mathrm{~Hz}$ ) until no heat signal could be detected anymore (usually up to $20 \mathrm{~h}$ ).

\section{Statistical analysis and baseline correction}

All nanocalorimetric experiments shown in the paper were performed 3 times. Heat flow $P(t)$ raw data was first filtered by an average filter to minimize the noise level. Mean values are indicated by lines on the figures and the shaded regions correspond to the standard error (SE), calculated using OriginLab ${ }^{\circledR}$ software. For baseline correction of the $P(t)$ curves, a spline fit was generated by the baseline correction function of the OriginLab ${ }^{\circledR}$ software (Fig. S7). Oxygen consumption and OD measurements were performed 3 times as well. Calibration of the oxygen sensor was performed according to a protocol provided by PyroScience $\mathrm{GmbH}$.

\section{Conclusions}

There is a general consensus among healthcare actors involved in infectious diseases management and microbiologists that the implementation of new technologies aiming to reduce the time to result of current clinical AST protocols is a key issue in combatting the increasingly important global health problem related to AMR of clinically important bacterial strains. In this regard, we developed a miniaturized isothermal nanocalorimetry platform (INCFAST) providing a direct method for measuring bacterial metabolic heat of $E$. coli. Considering bacterial heat production as a new phenotype opens the way to a more comprehensive understanding of fundamental metabolic processes, especially upon drug exposure. High heat detection sensitivity and signal stability enabled detection of bacterial growth in the microincubator under normal conditions after only $\sim 2-3$ hours. Eventually infection in clinical samples could be monitored on a similar time scale. The integrated fluidic design facilitates sample handling and the implementation of automated assay protocols. The versatility of the method was first demonstrated by analyzing metabolic heat profiles for various culture conditions over extended durations. More importantly, we could demonstrate that our nanocalorimetry platform has high potential for evaluating the efficacy and activity of antimicrobial compounds, in particular with respect to the implementation of fast AST protocols. Based on our experimental heat flow curves, AST could be performed in 5-6 hours using a fast-growing laboratory strain of $E$. coli as bacterial model. Heat flow profiles provided a detailed real-time insight of the dynamics of antimicrobial effects for all 3 antibiotics tested (ciprofloxacin, ampicillin and gentamicin). We observed notable lag phase extensions close to MICs and alterations of transient features in the heat flow curves. MIC values obtained with the INCfAST platform are consistent with accepted clinical values for $E$. coli in the case of ciprofloxacin and ampicillin. Gentamicin showed a slightly higher value range, most likely due to its oxygen-dependent activity. In contrast to the standard BMD method, the calorimetric approach does not rely on optical parameters, in principal raw patient samples could therefore be used, omitting culture steps for sample purification. Discrimination between growth/growth inhibition in the presence of antibiotics was possible on a time scale of only a few hours. In this view, we anticipate that with the present nanocalorimetry approach, AST assays can be performed on a faster time scale and with enhanced information content than current conventional clinical methods.

\section{Conflicts of interest}


The authors declare no conflict of interest.

\section{Author Contributions}

Y.L. designed and characterized the new nanocalorimetry platform and performed the microbiological assays. Y.L., T.L. and M.G. wrote or revised the paper. T.L. and M.G. supervised this work.

\section{Acknowledgements}

The authors gratefully acknowledge the support of EPFL-ATMX and EPFL-AFA workshops for the fabrication of the nanocalorimetry thermostats and the 3D-printed microincubators, respectively, as well as Peter Brühlmeier (EPFL-ACl workshop) for the fabrication of the PCB for the sensor chip. We thank Prof. John McKinney and Dr. Neeraj Dhar for useful discussions. This work was carried out within the framework of the EU project "New Diagnostics for Infectious Diseases" (http://www.nd4id.eu/). This project received funding from the European Union's Horizon 2020 program under the Marie Skłodowska-Curie grant agreement No 675412 .

\section{Notes and references}

1 L. Cantas, S. Q. A. Shah, L. M. Cavaco, C. M. Manaia, F. Walsh, M. Popowska, H. Garelick, H. Bürgmann and H. Sørum, Front. Microbiol.

2 R. Sugden, R. Kelly and S. Davies, Nat Microbiol, 2016, 1, 16187.

3 S. B. Zaman, M. A. Hussain, R. Nye, V. Mehta, K. T. Mamun and N. Hossain, Cureus, 2017, 9, e1403.

4 F. D. Lowy, The Journal of Clinical Investigation, 2003, 111, 10.

5 J. O'Neill, Tackling Drug-Resistant Infections Globally: Final Report and Recommendations. The Review on Antimicrobial Resistance, Chaired by Jim O'Neill, 2016.

6 H. Goossens, M. Ferech, R. V. Stichele and M. Elseviers, 2005, 365, 9.

7 A. van Belkum, G. Lüdke, J. G. Lisby, G. Kahlmeter, A. Mohess, K. Becker, J. P. Hays, N. Woodford, K. Mitsakakis, J. Moran-Gilad, J. Vila, H. Peter, J. H. Rex and Wm. M. Dunne, Nat Rev Microbiol, 2019, 17, 51-62.

8 J. M. Andrews, Journal of Antimicrobial Chemotherapy, 2001, 48, 5-16.

9 M. Balouiri, M. Sadiki and S. K. Ibnsouda, Journal of Pharmaceutical Analysis, 2016, 6, 71-79.

10 S. Puttaswamy, S. K. Gupta, H. Regunath, L. P. Smith and S. Sengupta, Arch Clin Microbiol, 2018, 9(3).

11 A. van Belkum and W. M. Dunne, Journal of Clinical Microbiology, 2013, 51, 2018-2024.

12 P. Athamanolap, K. Hsieh, L. Chen, S. Yang and T.-H. Wang, Analytical Chemistry, 2017, 89, 11529-11536.
13 Ö. Baltekin, A. Boucharin, E. Tano, D. I. Andersson and J. Elf, Proceedings of the National Academy of Sciences, 2017, 201708558.

14 O. Braissant, D. Wirz, B. Göpfert and A. U. Daniels, Sensors, 2010, 10, 9369-9383.

15 O. Braissant, A. Bachmann and G. Bonkat, Methods, 2015, 76, 27-34.

16 O. Braissant, D. Wirz, B. Göpfert and A. U. Daniels, FEMS Microbiology Letters, 2010, 303, 1-8.

17 A. Trampuz, S. Salzmann, J. Antheaume and A. U. Daniels, Transfusion, 2007, 47, 1643-1650.

18 U. von Ah, D. Wirz and A. U. Daniels, Journal of Clinical Microbiology, 2008, 46, 2083-2087.

19 D. Baldoni, H. Hermann, R. Frei, A. Trampuz and A. Steinhuber, Journal of Clinical Microbiology, 2009, 47, 774-776.

20 L. N. Yang, F. Xu, L. X. Sun, Z. B. Zhao and C. G. Song, J Therm Anal Calorim, 2008, 93, 417-421.

21 U. von Ah, D. Wirz and A. Daniels, BMC Microbiology, 2009, 9, 106.

22 J. Higuera-Guisset, J. Rodríguez-Viejo, M. Chacón, F. J. Muñoz, N. Vigués and J. Mas, Thermochimica Acta, 2005, 427, 187-191.

23 F. Buchholz, J. Lerchner, F. Mariana, U. Kuhlicke, T. R. Neu, H. Harms and T. Maskow, Biofouling, 2012, 28, 351362.

24 J. Lerchner, A. Wolf, F. Buchholz, F. Mertens, T. R. Neu, H. Harms and T. Maskow, Journal of Microbiological Methods, 2008, 74, 74-81.

25 F. Mariana, F. Buchholz, J. Lerchner, T. R. Neu, H. Harms and T. Maskow, International Journal of Medical Microbiology, 2013, 303, 158-165.

26 E. A. Johannessen, J. M. R. Weaver, L. Bourova, P. Svoboda, P. H. Cobbold and J. M. Cooper, Analytical Chemistry, 2002, 74, 2190-2197.

27 F. E. Torres, P. Kuhn, D. D. Bruyker, A. G. Bell, M. V. Wolkin, E. Peeters, J. R. Williamson, G. B. Anderson, G. P. Schmitz, M. I. Recht, S. Schweizer, L. G. Scott, J. H. Ho, S. A. Elrod, P. G. Schultz, R. A. Lerner and R. H. Bruce, PNAS, 2004, 101, 9517-9522.

28 M. I. Recht, F. E. Torres, D. D. Bruyker, A. G. Bell, M. Klumpp and R. H. Bruce, Analytical Biochemistry, 2009, 388, 204-212.

29 W. Lee, W. Fon, B. W. Axelrod and M. L. Roukes, Proceedings of the National Academy of Sciences, 2009, 106, 15225-15230.

30 N. Inomata, M. Toda, M. Sato, A. Ishijima and T. Ono, Applied Physics Letters, 2012, 100, 154104.

31 S. Wang, S. Yu, M. S. Siedler, P. M. Ihnat, D. I. Filoti, M. Lu and L. Zuo, Review of Scientific Instruments, 2016, 87, 105005.

32 J. Kim, S. Seo, J. Kim, S. Nam and W. Lee, 2019 IEEE 32nd International Conference on Micro Electro Mechanical Systems (MEMS), 544-546. 
33 I. Wadsö, D. Hallén, M. Jansson, J. Suurkuusk, T. Wenzler and O. Braissant, Thermochimica Acta, 2017, 652, 141149.

34 R. Krenger, T. Lehnert and M. A. M. Gijs, Lab on a Chip, 2018, 18, 1641-1651.

35 K. Stevenson, A. F. McVey, I. B. N. Clark, P. S. Swain and T. Pilizota, Sci Rep, 2016, 6, 38828.

36 E. Rosenberg and I. Zilber-Rosenberg, Gut Microbes, 2016, 7, 283-285.

37 EUCAST, The European Committee on Antimicrobial Susceptibility Testing. Routine and extended internal quality control for MIC determination and disk diffusion as recommended by EUCAST. Version 9.0, 2019. http://www.eucast.org., 2019.

38 P. D. Walker and S. V. Shah, American Journal of Physiology-Cell Physiology, 1987, 253, C495-C499.

39 M. Regué, B. Hita, N. Piqué, L. Izquierdo, S. Merino, S. Fresno, V. J. Benedí and J. M. Tomás, IAI, 2004, 72, 54-61.

40 J. H. Moffatt, M. Harper, P. Harrison, J. D. F. Hale, E. Vinogradov, T. Seemann, R. Henry, B. Crane, F. St. Michael, A. D. Cox, B. Adler, R. L. Nation, J. Li and J. D. Boyce, AAC, 2010, 54, 4971-4977.

41 L. Yang, J. A. J. Haagensen, L. Jelsbak, H. K. Johansen, C. Sternberg, N. Høiby and S. Molin, JB, 2008, 190, 27672776.

42 A. Harms, E. Maisonneuve and K. Gerdes, Science, 2016, 354, aaf4268.

43 I. Swinnen, International Journal of Food Microbiology, 2004, 94, 137-159.

44 M. D. Rolfe, C. J. Rice, S. Lucchini, C. Pin, A. Thompson, A. D. S. Cameron, M. Alston, M. F. Stringer, R. P. Betts, J. Baranyi, M. W. Peck and J. C. D. Hinton, Journal of Bacteriology, 2012, 194, 686-701.

45 B. Li, Y. Qiu, H. Shi and H. Yin, Analyst, 2016, 141, 30593067.

46 O. Fridman, A. Goldberg, I. Ronin, N. Shoresh and N. Q. Balaban, Nature, 2014, 513, 418-421.

47 W. F. Loomis and B. Magasanik, Journal of bacteriology, 1967, 93, 1397-1401.

48 A. Solopova, J. van Gestel, F. J. Weissing, H. Bachmann, B. Teusink, J. Kok and O. P. Kuipers, Proceedings of the National Academy of Sciences, 2014, 111, 7427-7432.

49 P. Johansson and I. Wadsö, Thermochimica Acta, 1999, 342, 19-29.

50 O. Braissant, G. Bonkat, D. Wirz and A. Bachmann, Thermochimica Acta, 2013, 555, 64-71.

51 M. A. Kohanski, D. J. Dwyer and J. J. Collins, Nature Reviews Microbiology, 2010, 8, 423-435.

52 J. B. Russell, J Mol Microbiol Biotechnol, 2007, 13, 1-11.

53 F. Buchholz, A. Wolf, J. Lerchner, F. Mertens, H. Harms and T. Maskow, Antimicrobial Agents and Chemotherapy, 2010, 54, 312-319.

54 J. H. Yang, S. N. Wright, M. Hamblin, D. McCloskey, M. A. Alcantar, L. Schrübbers, A. J. Lopatkin, S. Satish, A. Nili, B. O. Palsson, G. C. Walker and J. J. Collins, Cell, 2019, 177, 1649-1661.e9. 